\title{
Concordancia entre la radiografía panorámica y la tomografía computarizada de haz cónico en la relación de los terceros molares mandibulares con el conducto dentario inferior
}

\author{
Concordance between panoramic radiography and cone beam computed tomography in the relationship of mandib- \\ ular third molars with inferior dental canal
}

\begin{abstract}
Raúl Rafferty Herrera Mujica ${ }^{1, a}$, Liz Katty Ríos Villasis ${ }^{1, \mathrm{~b}}$, Roberto Antonio León Manco ${ }^{1, \mathrm{c}}$, Jorge Arturo Beltrán Silva ${ }^{1, \mathrm{~d}}$
\end{abstract}
\section{RESUMEN}

Objetivo: determinar la concordancia entre la radiografía panorámica y la tomografía computarizada de haz cónico en el análisis de la relación de los terceros molares mandibulares con el conducto dentario inferior en una población peruana. Material y métodos: la investigación fue observacional, descriptiva, transversal y retrospectiva. La muestra fue de 112 terceros molares y para la evaluación radiográfica se utilizó la clasificación modificada de Robert Langlais. Así mismo, otras variables fueron edad, sexo y lado. Las pruebas estadísticas empleadas fueron chi cuadrado, prueba U de Mann Whitney, prueba de Kruskall Wallis y el Índice de Kappa de Cohen. Resultados: según la radiografía panorámica, el 82,1\% (n=92) de los terceros molares presentó superposición, del cual el mayor porcentaje fue para el signo "discontinuidad" con $25,9 \%$ $(n=29)$. Según la tomografía computarizada de haz cónico, el 65,2\% $(n=73)$ de los molares presentó "contacto". El signo radiográfico "banda oscura + discontinuidad" tuvo una correspondencia del $88,9 \%(\mathrm{n}=24)$ con la presentación tomográfica de "contacto". Conclusiones: existe una baja concordancia diagnóstica entre la radiografía panorámica y la tomografía computarizada de haz cónico en el análisis de la relación del tercer molar mandibular con el conducto dentario inferior. Además, no existe diferencia estadísticamente significativa de esta relación con el sexo, ni con la edad y ni tampoco con el lado.

PALABRAS CLAVE: tercer molar, radiografía panorámica y tomografía.

\section{SUMMARY}

Objective: to determine the concordance between panoramic radiography and cone beam computed tomography in analysis of the relationship of mandibular third molars with inferior dental canal in patients attended in a peruvian population. Material and methods: this research was observational, descriptive, cross-sectional and retrospective. The sample was 112 mandibular third molars and modified classification of Robert Langlais was used for radiographic evaluation. Likewise, other variables were age, sex and side. The statistical tests used were chi square, Mann Whitney U test, Kruskall Wallis test and Cohen's kappa coefficient. Results: according to panoramic radiography, $82.1 \%(\mathrm{n}=92)$ of third molars showed superposition, of which the highest percentage was for "interruption of white line" with $25.9 \%(\mathrm{n}=29)$. According to cone beam computed tomography, $65.2 \%(\mathrm{n}=73)$ of the molars showed "contact". Radiographic sign "darkening of root + interruption of white line" had correspondence of $88.9 \%(n=24)$ with tomographic presentation of "contact". Conclusions: there is low diagnostic concordance between panoramic radiography and cone beam computed tomography in analysis of the relationship of mandibular third molar with inferior dental canal and there is also no statistically significant difference of this relationship with sex, neither with age nor with side.

KEYWORDS: third molar, panoramic radiography and tomography.

Facultad de Estomatología, Universidad Peruana Cayetano Heredia. Lima, Perú.

Docente de la sección de Imagenología Bucomaxilofacial.

Maestro en Estomatología

Maestro en Salud Pública.

Doctor en Estomatología. 


\section{INTRODUCCIÓN}

La exodoncia de los terceros molares es una de las actividades clínicas más frecuentes de la cirugía bucal. Los terceros molares mandibulares ocupan el primer lugar, en orden de frecuencia, dentro del grupo de dientes retenidos; se presentan más en mujeres que en hombres $(1,2)$, y la prevalencia puede alcanzar hasta el $82,5 \%$ en función de la población estudiada (3). La retención de los terceros molares inferiores puede llegar a ser 1,9 a 3 veces más frecuente que la de los terceros molares superiores $(1,4)$.

El tratamiento que se realiza en los terceros molares mandibulares retenidos es la exodoncia y puede estar asociada a dolor en la erupción, dolor a la masticación, caries radicular en la segunda molar, pérdida del hueso alveolar del segundo molar y/o pericoronaritis (5). En otros casos se opta por la exodoncia profiláctica de aquellos molares que no presentan ningún tipo de sintomatología. Aunque discutida, los defensores de este tratamiento sostienen que la exodoncia profiláctica de los terceros molares evita los posibles problemas que estos pueden causar en el futuro, que de aparecer generarían un mayor riesgo de complicaciones, costos y morbilidad en el paciente de mayor edad (6).

No es recomendable realizar la exodoncia del tercer molar sin obtener un diagnóstico imagenológico previo. Dentro de las imágenes radiográficas sugeridas está la panorámica, esto por su amplia cobertura; complementada, si es posible, con una periapical para una observación más detallada $(7,8)$. Sin embargo, un estudio retrospectivo con 400 casos demostró que la radiografía panorámica es inadecuada para la predicción de la lesión al nervio tras la exodoncia del tercer molar (9).

Es importante tener en cuenta que los exámenes imagenológicos no solo son importantes para realizar el diagnóstico. La correcta interpretación de los estudios por imagen marca el punto de partida para la toma de decisiones a la hora de la intervención quirúrgica y si es pertinente se solicitarán exámenes especializados como la tomografía computarizada, que es un estudio de gran aporte, ya que brinda información confiable y certera para el éxito de los procedimientos quirúrgicos de terceros molares $(10,11)$. En el campo de la odontología se utilizan con mayor frecuencia dos tipos de tomografía: la tomografía espiral multicorte y la tomografía computarizada de haz cónico (10).

La tomografía computarizada de haz cónico ha demostrado que establece con mayor claridad, que la radiografía panorámica, la relación entre el tercer molar y el conducto dentario $(12,13)$. En varios casos reafirma la relación entre la raíz y el conducto; en otros, la relación que se determina en la radiografía panorámica no es tal y las raíces aparecen alejadas del conducto dentario. La menor dosis de radiación, equivalente en ocasiones al de una radiografía panorámica, hacen que su aplicación en el ámbito maxilofacial se extienda rápidamente en detrimento de la tomografía espiral multicorte $(10,12,14,15)$.

El objetivo de este trabajo fue determinar la concordancia entre la radiografía panorámica y la tomografía computarizada de haz cónico en el análisis de la relación de la raíz de los terceros molares mandibulares con el conducto dentario inferior.

\section{MATERIAL Y MÉTODOS}

El estudio fue retrospectivo, observacional, transversal y descriptivo (16). Se obtuvo la aprobación del Comité Institucional de Ética en Investigación de la Universidad Peruana Cayetano Heredia y la autorización de la Dirección General del Instituto de Diagnóstico Maxilofacial para el acceso a la base de datos imagenológicos de los pacientes correspondientes al año 2016, manteniendo en anonimato la identidad de los pacientes.

Se evaluaron las radiografías panorámicas y tomografías computarizadas de haz cónico de 112 molares mandibulares de 62 pacientes de nacionalidad peruana entre los 18 y 25 años de edad que tenían un estadío de Nolla mayor o igual a 7. Se excluyeron del estudio a pacientes que presentaban variantes anatómicas, piezas supernumerarias, entidades patológicas en la región de los terceros molares mandibulares; así como, a terceros molares en posición transversal (bucolingual).

La calibración del observador fue con un especialista en Radiología Oral y Maxilofacial de 15 años de experiencia (gold standar) en el análisis de la relación de la raíz de los terceros molares con el conducto dentario inferior según la clasificación modificada 
de Robert Langlais (17) que describe hallazgos radiográficos a nivel del conducto dentario inferior y sugiere la posible relación del mismo con la raíz del tercer molar teniendo en cuenta la superposición o no entre estas estructuras anatómicas. Esta clasificación menciona los siguientes signos: "no superposición", "banda oscura", "adelgazamiento", "discontinuidad", "cambio de dirección" y la combinación entre dos o más de estos signos; haciendo un total de 16 signos radiográficos. Para la evaluación tomográfica se consideró las presentaciones de "contacto" y "no contacto" entre la raíz del tercer molar mandibular y el conducto dentario inferior. El índice de Kappa obtenido fue de 0.90 .

El método utilizado fue la observación estructurada mediante la inspección visual de las radiografías digitales y tomografías en condiciones ideales para la interpretación imagenológica; esto es un espacio adecuado, semioscuro y silencioso $(18,19,20)$. Las sesiones de visualización de las radiografías y tomografías se realizaron en horas de la mañana, considerando un descanso visual de 15 minutos por cada 2 horas de trabajo en el computador y cada 20 minutos se realizó una pausa fijando la mirada en un punto lejano $(21,22)$. Las sesiones de visualización se llevaron a cabo en una habitación tranquila con luz ambiental tenue y en una computadora Apple iMAC con una pantalla de 27 pulgadas y con una resolución de 5120 x 2880 . En cuanto al procesador es de 3.5 $\mathrm{GHz}$ Intel Core i5 4690 que posee una memoria de 16GB DDR3 SDRAM $1600 \mathrm{MHz}$ y gráficos $2 \mathrm{~GB}$ AMD Radeon R9M290X. Los datos y variables a evaluar se registraron en una Ficha de Registro. Las imágenes radiográficas de la base de datos fueron obtenidas con el equipo Planmeca ProMax 2D y las imágenes tomográficas con el equipo Planmeca ProMax 3D que posee un tamaño mínimo de vóxel de $0,075 \mathrm{~mm}$, ambos equipos pertenecientes a la marca finlandesa Planmeca. Las imágenes se evaluaron por medio del software Romexis.

Se realizó un análisis univariado de las variables obteniendo frecuencias absolutas y relativas; así como, la obtención de promedios y desviación estándar de la variable cuantitativa. Se efectuó también un análisis bivariado de las variables mediante la prueba de Chicuadrado y se emplearon las pruebas no paramétricas de U de Mann Whitney y Kruskall Wallis. Para determinar el grado de concordancia se realizó el
Índice de Kappa de Cohen. El presente estudio contó con un nivel de confianza de $95 \%$ y un $\mathrm{p}<0.05$. El programa estadístico usado fue el SPSS versión 24.0.

\section{RESULTADOS}

Se evaluaron 121 pacientes entre 18 a 25 años de edad, a los que, con fines diagnósticos ajenos a la investigación, se les realizó la adquisición de una radiografía panorámica y de una tomografía computarizada de haz cónico. De los 121 pacientes, 62 presentaban al menos un tercer molar inferior. Se evaluaron en total 112 terceros molares inferiores y su relación (superposición, no superposición, contacto y no contacto) con el conducto dentario inferior, 56 molares fueron de pacientes de sexo masculino y 56 molares del sexo femenino. Los terceros molares inferiores del lado izquierdo fueron $57 \mathrm{y}$ del lado derecho fueron 55 .

Según la prueba estadística de Kappa, la concordancia entre la radiografía panorámica y la tomografía computarizada de haz cónico en el análisis de la relación de los terceros molares mandibulares con el conducto dentario inferior fue baja, con un valor de 0,40 (tabla 1).

Mediante la radiografía panorámica, el 82,1\% (n=92) de los molares inferiores presentó "superposición" al conducto dentario y el mayor porcentaje fue para "discontinuidad" con $25,9 \%(\mathrm{n}=29)$. Según la tomografía el $65,2 \%(n=73)$ presentó "contacto" entre la raíz del tercer molar con el conducto dentario (tabla 2).

El signo radiográfico más frecuente fue "discontinuidad" con $25,9 \% \quad(n=29) \quad$ y tuvo correspondencia con la presentación tomográfica de "contacto" en 69\% $(n=20)$. En segundo lugar, el signo radiográfico más frecuente fue "banda oscura" con $25 \%(n=28)$ y una correspondencia del $60,7 \%(n=17)$ con "contacto". En tercer lugar, el signo radiográfico "banda oscura + discontinuidad" con $24,1 \%(n=27)$ y una correspondencia de $88,9 \%(n=24)$ con la presentación tomográfica de "contacto" (tabla 3). 
Tabla 1. Concordancia entre la radiografía panorámica y la tomografía de haz cónico en el análisis de la relación de los terceros molares mandibulares con el conducto dentario inferior.

\begin{tabular}{llcccccccc}
\hline Panorámica & \multicolumn{1}{c}{ Contacto } & \multicolumn{9}{c}{ No contacto } & \multicolumn{2}{c}{ Total } & \multirow{2}{*}{ Kappa } \\
& \multicolumn{1}{c}{ n } & $\mathbf{\%}$ & $\mathbf{n}$ & $\mathbf{\%}$ & $\mathbf{n}$ & $\mathbf{\%}$ & & \\
Pan/Tom & & & & & & & \\
\hline & Superposición & 69 & 75 & 23 & 25 & 92 & 82,1 & \\
& No superposición & 4 & 20 & 16 & 80 & 20 & 17,9 & 0,4 \\
& Total & 73 & 65,2 & 39 & 34,8 & 112 & 100 & \\
\hline
\end{tabular}

Tabla 2. Frecuencia de la relación del tercer molar mandibular con el conducto dentario inferior mediante radiografías panorámicas y tomografías de haz cónico

\begin{tabular}{|c|c|c|c|}
\hline & Diagnósticos & $\mathbf{n}$ & $\%$ \\
\hline \multicolumn{4}{|l|}{ Panorámica } \\
\hline & No superposición & 20 & 17,9 \\
\hline & Banda oscura & 28 & 25,0 \\
\hline & Cambio de dirección & 1 & 0,9 \\
\hline & Discontinuidad & 29 & 25,9 \\
\hline & Adelgazamiento & 0 & 0,0 \\
\hline & Banda oscura + Cambio de dirección & 1 & 0,9 \\
\hline & Banda oscura + Discontinuidad & 27 & 24,1 \\
\hline & Banda oscura + Adelgazamiento & 0 & 0,0 \\
\hline & Cambio de dirección + Discontinuidad & 0 & 0,0 \\
\hline & Cambio de dirección + Adelgazamiento & 1 & 0,9 \\
\hline & Discontinuidad + Adelgazamiento & 3 & 2,7 \\
\hline & Banda oscura + Cambio de dirección + Discontinuidad & 0 & 0,0 \\
\hline & Banda oscura + Cambio de dirección + Adelgazamiento & 0 & 0,0 \\
\hline & Banda oscura + Discontinuidad + Adelgazamiento & 1 & 0,9 \\
\hline & Cambio de dirección + Discontinuidad + Adelgazamiento & 0 & 0,0 \\
\hline & Banda oscura + Cambio de dirección + Discontinuidad + Adelgazamiento & 1 & 0,9 \\
\hline
\end{tabular}

Panorámica

Superposición

No superposición

Tomografía

n: Frecuencia absoluta.

$\%$ : Frecuencia relativa. 
Tabla 3. Frecuencia de correspondencia entre la radiografía panorámica y la tomografía de haz cónico en la relación del tercer molar mandibular con el conducto dentario inferior

\begin{tabular}{lccccc}
\hline \multicolumn{1}{c}{ Panorámica } & \multicolumn{4}{c}{ Tomografía } \\
& Contacto & $\begin{array}{c}\text { No con- } \\
\text { tacto }\end{array}$ \\
& n & \% & n & \% \\
\hline No superposición & 4 & 20,0 & 16 & 80,0 \\
Banda oscura & 17 & 60,7 & 11 & 39,3 \\
Cambio de dirección & 1 & 100,0 & 0 & 0,0 \\
Discontinuidad & 20 & 69,0 & 9 & 31,0 \\
Adelgazamiento & 0 & 0,0 & 0 & 0,0 \\
Banda oscura + Cambio de dirección & 1 & 100,0 & 0 & 0,0 \\
Banda oscura + Discontinuidad & 24 & 88,9 & 3 & 11,1 \\
Banda oscura + Adelgazamiento & 0 & 0,0 & 0 & 0,0 \\
Cambio de dirección + Discontinuidad & 0 & 0,0 & 0 & 0,0 \\
Cambio de dirección + Adelgazamiento & 1 & 100,0 & 0 & 0,0 \\
Discontinuidad + Adelgazamiento & 3 & 100,0 & 0 & 0,0 \\
Banda oscura + Cambio de dirección + Discontinuidad & 0 & 0,0 & 0 & 0,0 \\
Banda oscura + Cambio de dirección + Adelgazamiento & 0 & 0,0 & 0 & 0,0 \\
Banda oscura + Discontinuidad + Adelgazamiento & 1 & 100,0 & 0 & 0,0 \\
Cambio de dirección + Discontinuidad + Adelgazamiento & 0 & 0,0 & 0 & 0,0 \\
Banda oscura + Cambio de dirección + Discontinuidad + Adelgazamiento & 1 & 100,0 & 0 & 0,0 \\
\hline
\end{tabular}

n: Frecuencia absoluta.

$\%$ : Frecuencia relativa.

\section{DISCUSIÓN}

Muchos estudios han investigado la relación anatómica entre el tercer molar mandibular y el conducto dentario inferior. La radiografía panorámica se utiliza con frecuencia en la planificación del tratamiento para la cirugía del tercer molar. Por lo tanto, existe la necesidad de un método simple y confiable para predecir la parestesia del nervio alveolar inferior mediante la evaluación de radiografías panorámicas. Varios estudios han propuesto factores de riesgo basados en hallazgos de imágenes panorámicas; sin embargo, aún no se han establecido factores predictivos confiables (23).

Tantanapornkul et al., evaluaron la precisión diagnóstica de la tomografía computarizada de haz cónico en comparación con las imágenes panorámicas en la predicción de la exposición del conducto dentario inferior durante la extracción de los terceros molares mandibulares impactados y concluyeron que la tomografia computarizada de haz cónico fue significativamente superior a las imágenes panorámicas (13). Por otro lado, Mela et al., con los resultados de su estudio demostraron la eficacia de la radiografía panorámica en la evaluación preoperatoria de los terceros molares mandibulares, ya que encontraron una correlación significativa entre este examen radiográfico y la tomografía (24). Mencionan también que la "discontinuidad", asociada o no "banda oscura", es indicativa de riesgo de "contacto" entre la raíz y el conducto dentario inferior, siendo este signo radiográfico sugerente de una exploración tomográfica. En el presente estudio, según la prueba estadística de Kappa, la concordancia diagnóstica entre la radiografía panorámica y la tomografía computarizada de haz cónico fue baja, esto quiere decir que no es lo mismo analizar la relación 
entre el tercer molar y el conducto dentario mediante la radiografía panorámica que por medio de la tomografía; sin embargo, cabe señalar que la asociación entre "banda oscura + discontinuidad" fue la que con más frecuencia mostró evidencias de "contacto" mediante la tomografía. La discrepancia entre los resultados de la presente investigación con los de Mela et al., puede deberse a que estos últimos analizaron una muestra de menor tamaño (50 molares), evaluaron únicamente raíces en "superposición", consideraron un rango etario diferente y utilizaron un tomógrafo con un tamaño de vóxel tres veces mayor (24).

En cuanto a los signos radiográficos, se mencionó que "discontinuidad" fue el que señalaba mayor frecuencia de compromiso del conducto dentario inferior según Mela et al (24); pero Tantanapornkul et al., consideran que el signo "banda oscura" es indicativo de una estrecha relación entre la raíz y la mencionada estructura anatómica; fue mediante la tomografía computarizada de haz cónico que evidenciaron que el "adelgazamiento o perforación" de la cortical del conducto se correlaciona significativamente con este signo radiográfico (25).

El presente estudio encuentra que la "banda oscura + discontinuidad" fue el signo que se evidenció con mayor frecuencia de correspondencia $(88,9 \%)$ con la presentación tomográfica de "contacto". Así mismo, se encontró que el $80 \%$ de los casos de "no superposición" presentaron "no contacto" y que el $75 \%$ de los casos de "superposición" presentaron "contacto". Por otro lado, Aranda concluye que, de 577 terceros molares evaluados mediante radiografías panorámicas, el 59 \% presenta "no superposición" con el conducto dentario inferior, mientras que el $41 \%$ presenta "superposición" (17). De los 237 molares que presentaron superposición, según la clasificación modificada de Langlais, el signo radiográfico más frecuente fue "adelgazamiento + discontinuidad".

La investigación de Aranda (17), fue punto de partida para que se realicen estudios similares por parte de odontólogos de países como Ecuador, Colombia, Bolivia, Costa Rica y México; los mismos que evaluaron los signos radiográficos según la clasificación modificada de Robert Langlais. Alanoca (26), Agrada (27), Serna (28), Bastidas (29), Pineda (30) y Villavicencio (31); refieren haber encontrado mayor frecuencia de "superposición" y los signos radiográficos más frecuentes fueron: "banda oscura + discontinuidad", "banda oscura + cambio de dirección", "discontinuidad", "banda oscura", "banda oscura + discontinuidad + adelgazamiento" y "banda oscura + discontinuidad"; respectivamente.

Es importante mencionar que los investigadores coincidieron en sugerir que se realicen estudios utilizando la tomografía computarizada de haz cónico con el fin de determinar la correspondencia entre cada signo radiográfico bidimensional con la respectiva presentación tomográfica tridimensional y que fueron estas sugerencias las que incentivaron la ejecución de la presente investigación; la cual, a nivel radiográfico, coincide con estos investigadores en que hubo mayor frecuencia de "superposición" y que, al igual que Serna (28), el signo radiográfico más observado fue "discontinuidad".

Con respecto a la edad, diversos estudios tomaron en cuenta diferentes rangos etarios que oscilan desde 18 a 74 años; pero cabe mencionar que, a mayor edad, mayor dificultad para visualizar, en la radiografía panorámica, los signos propuestos por Langlais (32), ya que con el paso de los años el hueso tiende a perder densidad y las raíces de los dientes tienden a incrementar su radiopacidad por la aposición de cemento en su superficie. En el presente estudio no se encontró diferencia estadísticamente significativa, lo cual indica que no se encontró asociación entre la relación de las raíces del tercer molar y el conducto dentario inferior con la edad, posiblemente este resultado sea consecuencia de que el grupo etario de esta investigación oscila entre los 18 y 25 años. Con respecto al sexo, Mela et al., en base a la investigación que realizaron en 50 terceros molares inferiores, mencionan que hubo diferencia estadísticamente significativa entre los signos radiográficos y el sexo; siendo "banda oscura" el único signo que mostró tal asociación (24). Así mismo, mediante la tomografía, encontraron que hubo mayor frecuencia de "no contacto" con el conducto dentario inferior en el sexo masculino; sin embargo, no hubo diferencia estadísticamente significativa. En la presente investigación, no se encontró asociación con respecto al sexo, ni por medio de la evaluación radiográfica ni tomográfica, coincidiendo en que se evidenció mayor frecuencia de "no contacto" con el conducto dentario inferior en el sexo masculino. 
Estos resultados pueden explicarse por la conocida teoría de la reducción terminal dentaria de Adloff, que señala la futura desaparición del tercer molar en la especie humana, aspecto evolutivo consecuencia de la disminución de la actividad masticatoria que junto a una mayor frecuencia de agenesia de terceros molares en mujeres con respecto a varones, con una relación de 3 a 2; conllevarían a una reducción del tamaño mandibular incitando así el contacto de las raíces del tercer molar con la cortical del conducto dentario inferior $(33,34,35)$. En cuanto al lado, es importante señalar que el triángulo retromolar derecho suele ser mayor que el izquierdo, por lo cual, los accidentes correspondientes a la erupción de la muela del juicio son más frecuentes en el lado izquierdo que en el derecho, debido a que tiene menos espacio para erupcionar (36). Se puede mencionar que al revisar la literatura son varias investigaciones que no señalan el análisis de la relación del tercer molar con el conducto dentario y su asociación con el lado; sin embargo, eso no indica que no la hayan tomado en cuenta.

La presente investigación discrepa con Serna (28) y concluye que no hay diferencia estadísticamente significativa entre el lado y la relación de las raíces del tercer molar y el conducto dentario inferior.

En conclusión, existe una baja concordancia entre la radiografía panorámica y la tomografía computarizada de haz cónico en el análisis de la relación del tercer molar mandibular con el conducto dentario inferior. Además, no existe diferencia estadísticamente significativa de esta relación con el sexo, ni con la edad y ni tampoco con el lado. El signo radiográfico "banda oscura + discontinuidad"; presentó una frecuencia de correspondencia con la presentación tomográfica de "contacto" en $88,9 \%$.

\section{Correspondencia:}

Raúl Rafferty Herrera Mujica

Correo electrónico: raul.herrera.m@upch.pe

\section{REFERENCIAS BIBLIOGRÁFICAS}

1. Hashemipour MA, Tahmasbi-Arashlow M, FahimiHanzaei F. Incidence of impacted mandibular and maxillary third molars: a radiographic study in a Southeast Iran population. Med Oral Patol Oral Cir Bucal. 2013;1;18(1):140-5.

2. Padhye MN, Dabir AV, Girotra CS, Pandhi VH. Pattern of mandibular third molar impaction in the Indian population: a retrospective clinico-radiographic survey. Oral Surg Oral Med Oral Pathol Oral Radiol. 2013;116(3):161-6.

3. Chu FC, Li TK, Lui VK, Newsome PR, Chow RL, Cheung LK. Prevalence of impacted teeth and associated pathologies - a radiographic study of the Hong Kong Chinese population. Hong Kong Med J. 2003; 9: 58-63.

4. Quek SL, Tay CK, Tay KH, Toh SL, Lim KC. Pattern of third molar impaction in a Singapore Chinese population: a retrospective radiographic survey. Int J Oral Maxillofac Surg. 2003; 32: 548-52.

5. Medeiros PJ. Cirugía de dientes incluídos: Extracción del tercer molar. Sao Paulo: Livraria Santos Editora Limitada; 2006.

6. Kandasamy S, Rinchuse DJ, Rinchuse DJ. The wisdom behind third molar extractions. Aust Dent J. 2009;54 (4): 284-92.

7. Rood JP, Shehab BA. The radiological prediction of inferior alveolar nerve injury during third molar surgery. Br J Oral Maxillofac Surg. 1990;28(1):20-5.

8. Manotas I. Exodoncia del tercer molar: Factores que determinan complejidad. Duazary. 2008; 5(2): 141-7.

9. Szalma J, Lempel E, Jeges S, Szabó G, Olasz L. The prognostic value of panoramic radiography of inferior alveolar nerve damage after mandibular third molar removal: retrospective study of 400 cases. Oral Surg Oral Med Oral Pathol Oral Radiol Endod. 2010; 109: 294-302.

10. Lopez J, Vergara M, Rudolph M, Guzmán CL. Prevalencia de variables anatómicas en el recorrido de los conductos mandibulares. Estudio mediante tecnología cone beam. Rev Fac Odontol Univ Antioq. 2010; 22 (1): 23-32.

11. Ruge OY, Camargo OA, Ortiz YP. Consideraciones anatómicas del conducto alveolar inferior. Revista Facultad de Odontología Universidad de Antioquia. 2009. 21(1):86-97.

12. Umar G, Obisesan O, Bryant C, Rood JP. Elimination of permanent injuries to the inferior alveolar nerve following surgical intervention of the "high risk" third molar. Br J Oral Maxillofac Surg. 2013;51(4):353-7.

13. Tantanapornkul W, Okouchi K, Fujiwara Y, et al. A comparative study of cone-beam computed tomography and conventional panoramic radiography in assessing the topographic relationship between the mandibular canal and impacted third molars. Oral Surg Oral Med Oral Pathol Oral Radiol Endod. 2007; 103: 253-9.

14. Jhamb A, Dolas RS, Pandilwar PK, Mohanty S. Comparative efficacy of spiral computed tomography and orthopantomography in preoperative detection of relation of inferior alveolar neurovascular bundle to the impacted mandibular third molar. J Oral Maxillofac Surg. 2009; 67: 58-66.

15. Friedland M, Donoff B, Dodson TB. The use of 
3-dimensional reconstructions to evaluate the anatomic relationship of the mandibular canal and impacted mandibular third molars. J Oral Maxillofac Sur. 2008; 66(8):1678-85.

16. Hernández R, Fernández C, Baptista P. Metodología de la Investigación. 6ta ed. Ciudad de México: Mc Graw Hill; 2014.

17. Aranda L. Frecuencia de la proximidad del conducto dentario inferior con las raíces de las terceras molares en pacientes de la clínica dental de la Universidad Peruana Cayetano Heredia entre los años 2008-2010. Tesis para optar por el título de Cirujano Dentista. Lima: Universidad Peruana Cayetano Heredia; 2012.

18. Mallya S, Lam E. White and Pharoah's Oral Radiology. Principles and Interpretation. 8va ed. St. Louis: Elsevier; 2019.

19. Sarment D. Tomografía computarizada de haz cónico: Diagnóstico, aplicaciones oral y maxilofacial. Caracas: Amolca; 2017.

20. García A, Viteri G, Saiz R, Broncano J, Dámaso J. Ergonomía del puesto de trabajo en radiología. Radiología. 2011; 53 (6): 507-515.

21. Vertinsky T, Forster B. Prevalence of eye strain among radiologists: influence of viewing variables on symptoms. AJR. 2005; 184:681-86.

22. Echevarri S, Giraldo D, Lozano L, Mejia P, Montoya L, Vasquez E. Síndrome de visión por computador: una revisión de sus causas y del potencial de prevención. Rev CES Salud Publica. 2012;3(2):193-201.

23. Nakamori K, Fujiwara K, Miyazaki A, et al. Clinical assessment of the relationship between the third molar and the inferior alveolar canal using panoramic images and computed tomography. J Oral Maxillofac Surg. 2008; 66: 2308-13.

24. Mela OA, Tawfik MAM, Mansour NA. Assessment of the relationship between the mandibular canal and impacted third molars using cone beam computed tomography. Mansoura J Dent. 2014;1(3):49-55.

25. Tantanapornkul W, Okochi K, Bhakdinaronk A, Ohbayashi N, Kurabayashi T. Correlation of darkening of impacted mandibular third molar root on digital panoramic images with cone beam computed tomography findings. Dentomaxillofac Radiol. 2009; 38:11-16.

26. AlanocaA. Frecuencia de la localización de los terceros molares en radiografías panorámicas de pacientes de 18 a 35 años, que acuden a la Caja Petrolera de Salud en La Paz, Bolivia durante el periodo 20122013. Tesis para optar por al título de Especialista en Radiología Bucal y Maxilofacial. Lima: Universidad Peruana Cayetano Heredia; 2016.

27. Agrada B. Frecuencia de la localización de terceras molares y proximidad al CDI en radiografías panorámicas de pacientes atendidos en el servicio de Radiología de la Clínica privada de la ciudad de
Ayacucho durante los años 2014 y 2015. Tesis para optar al título de Especialista en Radiología Bucal y Maxilofacial. Lima: Universidad Peruana Cayetano Heredia; 2016.

28. Serna L. Frecuencia de la localización de las terceras molares en radiografías panorámicas de pacientes atendidos en un centro radiológico privado de la ciudad de Ambato, Tungurahua -Ecuador durante el periodo 2013-2014. Tesis para optar por al título de Especialista en Radiología Bucal y Maxilofacial. Lima: Universidad Peruana Cayetano Heredia; 2016.

29. Bastidas V. Frecuencia de la localización de terceros molares al conducto dentario inferior en radiografías panorámicas de pacientes atendidos en un centro radiológico privado oral y maxilofacial de la ciudad de Ipiales Nariño, Colombia durante los años 2013 y 2014. Tesis para optar al título de Especialista en Radiología Bucal y Maxilofacial. Lima: Universidad Peruana Cayetano Heredia; 2016.

30. Pineda Z. Frecuencia de la localización de los terceros molares y la proximidad al conducto dentario inferior en radiografías panorámicas en pacientes atendidos en el Centro de Radiología e Imagen Maxilofacial de la ciudad de Cuautitlán Izcalli, México durante los años 2013 y 2014. Tesis para optar al título de Especialista en Radiología Bucal y Maxilofacial. Lima: Universidad Peruana Cayetano Heredia; 2016.

31. Villavicencio M. Frecuencia de la localización de las terceras molares en radiografías panorámicas de pacientes atendidos en el Centro Radiológico Tomodent, Guanacaste - Costa Rica, 2015. Tesis para optar al título de Especialista en Radiología Bucal y Maxilofacial. Lima: Universidad Peruana Cayetano Heredia; 2017.

32. Langlais R, Langland O, Nortjé C. Diagnostic imaging of the jaws. Baltimore: Williams\& Wilkins; 1995.

33. Arboleda LA, Echeverri J, Restrepo LA, Marín ML, Vásquez G, Gómez JC, Manco HA, Pérez CM, Taborda E. Agenesia dental. Revisión bibliográfica y reporte de dos casos clínicos. Rev Fac. Odontol Univ Antioq. 2006; 18(1):47-54.

34. Botina CJ, Rodríguez LF, Cepeda EC, Zabala DF, González Colmenares G. Frecuencia de agenesias de terceros molares: relación con el tamaño mandibular. Rev Nac Odontol. 2012; 8(15): 52-6.

35. Colorado ML, Huitzil E. Agenesia de terceros molares en pacientes del Centro del estado de Veracruz. Rev Tamé. 2015; 4 (11): 379-82.

36. Velayos JL, Santana H. Anatomía de la cabeza para odontólogos. $4^{\mathrm{a}}$ ed. Buenos Aires: Editorial Médica Panamericana; 2007.

Recibido: 04-02-20

Aceptado: 15-06-20 